\title{
Hybrid Control of Bifurcation and Chaos in Dynamic Model of Compound TCP under RED
}

\author{
Mengya Geng1, Dawei Ding2*, Han Wang1, Jinyang Huang1', Jingwei Wang1, Haiyan Wang1 \\ ${ }^{1}$ School of Electronics and Information Engineering, Anhui University, Hefei, 230000, China. \\ 2 Key Lab. of Intelligent Computing and Signal Processing, Ministry of Education, Anhui University, Hefei \\ 230000, China. \\ * Corresponding author. Email: dwding@ahu.edu.cn \\ Manuscript submitted May 25, 2015; accepted August 8, 2015. \\ doi: 10.17706/ijcce.2016.5.3.186-195
}

\begin{abstract}
Compound TCP(CTCP) is a practical congestion control algorithm for high-speed and long delay networks. In this paper we address the problem of bifurcation and chaos analysis and its control of a nonlinear congestion control model with CTCP connections and random early detection (RED) gateway. First, we briefly analyze the nonlinear dynamics of the CTCP network with respect to system parameters. Then a hybrid control strategy using both state feedback and parameter perturbation is employed to control the bifurcation and stabilize the chaotic orbits embedded in this discrete-time dynamic system of CTCP/RED. Theoretical analysis show that the controlled system can achieve a stable average queue size in an extended range of parameters and even completely eliminates the chaotic behavior in a particular range of parameters. Therefore it is possible to decrease the sensitivity of RED parameters. Finally, numerical simulations are provided to show the effectiveness of the theoretical results.
\end{abstract}

Key words: Chaos control, dynamical systems, congestion control, bifurcation, CTCP, RED.

\section{Introduction}

Active Queue Management (AQM) schemes, which aim to provide timely indications of incipient congestion in the network to end-systems, also play an important role in Quality of Service (QoS) [1]. The Transmission Control Protocol (TCP) is responsible for regulating traffic of end-systems in the Internet [2]. Random Early Detection (RED) is one of the most prominent congestion avoidance schemes in the Internet routers [3]. It is reported that the interaction between TCP and RED often leads to undesirable behaviors, such as bifurcation and chaos, which may degrade network performance [4], [5]. As it is demonstrated by K. Tan, J. M. Song, Q. Zhang and M. Sridharan [6] that a TCP/RED system could be approximately modeled as a nonlinear map which exhibits a rich variety of irregular behaviors such as bifurcation and chaos.

The stability of TCP Reno/RED system has been studied in [7], [8] and it is shown that chaotic dynamics with respect to the variability of RED parameters [9], [10]. L. Chen, X. F. Wang and Z. H. Zheng [11], [12] investigated the possibility of controlling bifurcation and chaos in the TCP/RED system by several time delayed feedback control strategies. X. S. Luo, G. Chen, G. Wang, B. H. Fang and J. Qing [13] designed a hybrid control strategy to control the bifurcation and chaos in a discrete nonlinear dynamical system. D. W. Ding, J. Zhu and X. S. Luo [14] applied this hybrid control strategy into the congestion control system and put off the occurrence of bifurcation successfully. 
However the initial TCP versions developed for cable networks were not adequate any more. Moving bulk data quickly over high-speed data network is a requirement for many applications [6]. With time delayed feedback, stability and bifurcation phenomena become important considerations for performance evaluation [15]. Compound TCP, as a new congestion control protocol for high-speed and long delay networks, is a combination of both delay-based and loss-based congestion avoidance approach. CTCP is a promising algorithm to achieve high link utilization whereas maintaining good RTT fairness and TCP fairness [6].

In this paper, in order to study the problem of bifurcation and chaos control of CTCP under RED congestion model, first we have briefly introduce the dynamic one-order discrete equation to describe the CTCP under RED. Then, by theoretical analysis, we derive that the fixed point of the system is positive real solution of a polynomial. Meanwhile, a stable range of a system parameter is analyzed and the existence of period doubling bifurcation and chaos behavior is confirmed by bifurcation diagram and Lyapunov exponent diagram as well. Finally, in order to extend the stable range of parameter in CTCP under RED, efforts are made to control the bifurcation and to stabilize the chaotic orbits to the fixed point in the system through the hybrid control strategy. Theoretical analysis and numerical simulations are given to verify the effectiveness of the results.

The remainder of this paper is organized as follows. Presented in Section 2 is the dynamical model of CTCP under RED system. Then in the third section, the fixed point of the model is analyzed and the bifurcation and chaotic behaviors of the system are shown by bifurcation diagram and Lyapunov exponent diagram. In Section 4, the proposed hybrid control strategy is applied to stabilizing the chaotic orbits of the system. Finally, the conclusion of this paper is given in Section 5.

\section{Dynamic Model of CTCP}

In this section, we briefly introduce the dynamic model of a CTCP under RED system. The average queue size is employed as the state variable in the model. The network topology and other conditions are the same as those in [11]. The final dynamic model of the CTCP under RED system is as follows:

$$
\bar{q}_{k+1}=\left\{\begin{array}{l}
(1-w) \bar{q}_{k}, \text { if } \bar{q}_{k}<\bar{q}_{l} \\
(1-w) \bar{q}_{k}+w B, \text { if } \bar{q}_{k}<\bar{q}_{l} \\
(1-w) \bar{q}_{k}+\left(\frac{N}{\alpha^{\frac{1}{2-k}}\left(1-(1-\beta)^{1-k}\right) p^{\frac{1}{2-k}}}\left[\frac{1-(1-\beta)^{\frac{1-k}{2-k}}}{2-k}\right]^{\frac{1-k}{2-k}} \times-\frac{C d}{M}\right) \\
\text { otherwise }
\end{array}\right)
$$

where the variables are listed below:

$q_{k+1}$ : average queue size in round $k+1$;

$q_{k}$ : average queue size in round $k$;

$p_{k}$ : drop probability in round $k$;

$w$ : exponential averaging weight in RED;

$N$ : number of TCP connections;

$C$ : capacity of the link between the two routers;

$d$ : round-trip propagation delay;

$M$ : packet size;

$B$ : buffer size of RED router. 
The drop probability in round $k$ is

$$
p_{k}=\left\{\begin{array}{l}
0, \text { if } \bar{q}_{k}<q_{\min } \\
1, \text { if } \bar{q}_{k}>q_{\max } \\
\frac{\bar{q}_{k}-q_{\min }}{q_{\max }-q_{\min }} p_{\max }, \text { otherwise }
\end{array}\right.
$$

where $q_{\min }$ is the minimum threshold and $q_{\max }$ is the maximum threshold.

\section{Fixed Point and Bifurcation}

Equation (1) is denoted as

$$
\bar{q}_{k+1}=g\left(\bar{q}_{k}, \rho\right)
$$

where $\rho$ summarized the system parameters, including the exponential averaging weight $w$, the maximum packet drop probability $p_{\max }$, the minimum threshold $q_{\min }$ and the maximum threshold $q_{\max }$. if an average queue size satisfies the equation

$$
q^{*}=g\left(q^{*}, \rho\right)
$$

Then $q^{*}$ is a fixed point of mapping $g($.

We should choose the system parameter appropriately in order that the fixed point of system remains between $q_{\min }$ and $q_{\max }$. Therefore the fixed point $q^{*}$ is given as the real solution of the following polynomial:

$$
y-e \cdot\left(y-q_{\min }\right)^{\frac{-1}{2-k}}-\frac{C d}{M}=0
$$

where

$$
e=N\left[\frac{1-(1-\beta)^{\frac{1-k}{2-k}}}{2-k}\right]^{\frac{1-k}{2-k}}\left(\alpha^{\frac{1}{2-k}}\left(1-(1-\beta)^{1-k}\right)\left(\frac{p_{\max }}{q_{\max }-q_{\min }}\right)^{\frac{1}{2-k}}\right)^{-1}
$$

From (5), we know that the fixed point does not depend on the exponential averaging weight $w$. In order to analyze the stability of the fixed point $q^{*}$, we consider the eigenvalue of the system

$$
\left.\frac{\partial g\left(\bar{q}_{k}, \rho\right)}{\partial \bar{q}_{k}}\right|_{\bar{q}_{k}=q^{*}}=1-w-w \frac{e}{2-k}\left(q^{*}-q_{\min }\right)^{\frac{k-3}{2-k}}
$$

The linear stability of the fixed point $q^{*}$ is 


$$
\left|1-w-w \frac{e}{2-k}\left(q^{*}-q_{\min }\right)^{\frac{k-3}{2-k}}\right|<1
$$

Changing the parameter setting can lead to the loss of stability of the fixed point if inequality (7) is not satisfied. In order to demonstrate the existence of such a bifurcation leading to the chaotic behavior of system, we choose the exponential averaging weight $w$ as the distinguished bifurcation parameter, its critical as (6) is a descending function of $w$. The critical value of $w$ can be deduced from which the eigenvalue given by (6) is 0.296 . The fixed point of system is stable when $w_{c}$ as

$$
w_{c}=\frac{2}{1+\frac{e}{2-k}\left(q^{*}-q_{\min }\right)^{\frac{k-3}{2-k}}}
$$

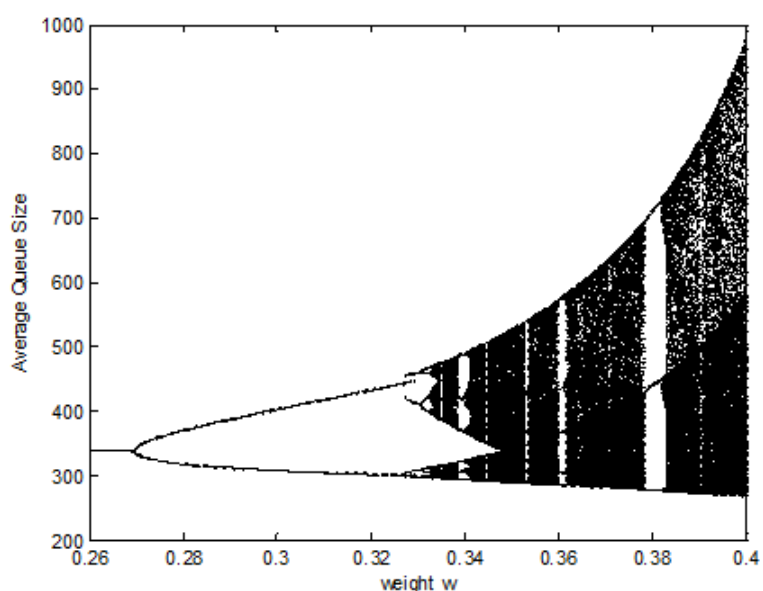

Fig. 1. Bifurcation diagram with as $w$ a parameter.

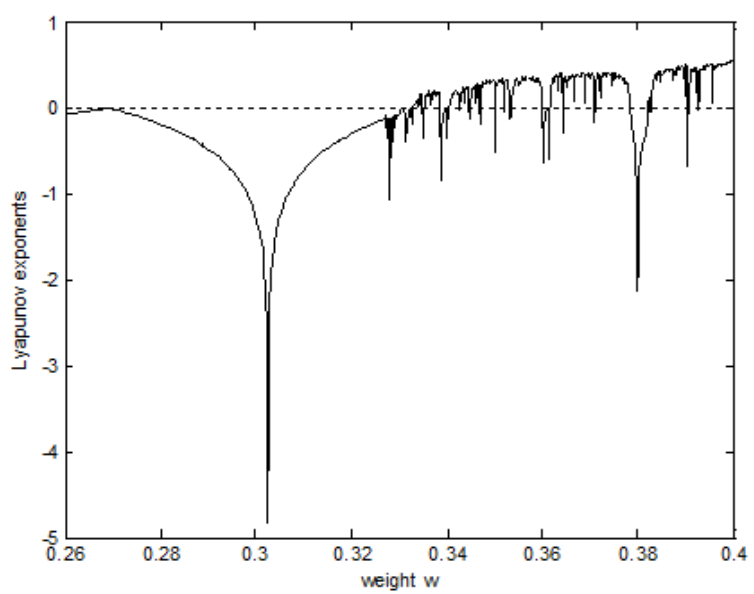

Fig. 2. Lyapunov exponent.

For example, we set system parameters as follows:

$q_{\max }=750$ packets; $q_{\min }=250$ packets; $p_{\max }=0.1 ; C=15 \mathrm{Mbit} / \mathrm{s} ; B=3750$ packets; $M=4000$ bit; $d=0.1 \mathrm{~s} ; N=1$; and we choose $k=0.75, \alpha=1 / 8$ and $\beta=1 / 2$.

From theoretical analysis in Sect. 3, it is drawn that the fixed point of the system $q^{*}=338.3$, The bifurcation diagram is plotted in Fig. 1, with $w$ varying from 0.26 to 0.40 . The bifurcation diagram is shown in Fig. 1. From Fig. 1, one can see that for $w<w_{c}$, the system is stable and these plots have a fixed point. When $w>w_{c}$, the system loses its stability and a super-critical period doubling bifurcation emerges. Lyapunov exponents are plotted in Fig. 2, we can see that when $w$ is small the exponent is negative, which corresponds to the single fixed point. As $w$ increases, the exponent slowly increases to zero and then becomes negative again due to a stable period-doubling orbit. Finally, it becomes positive and indicates that the system is in chaotic orbits. Both of the two diagrams show that the system enters into chaos state through a period-doubling route and some small periodic windows are embedded in the chaotic region.

\section{Control Bifurcation and Chaos}

In this section, we employ a hybrid control strategy proposed in [13] for controlling bifurcation and chaos in a stroboscopic model of TCP/RED. The objective is to extend the range of RED parameters, in which the system remains its stable dynamical behavior, and to delay or even eliminate the bifurcation 
completely. Now we apply both parameter perturbation and state feedback to the system; therefore we acquire the following controlled form of the map (3):

$$
\bar{q}_{k+m}=r g^{(m)}\left(\bar{q}_{k}, \rho\right)+(1-r) \bar{q}_{k}
$$

where $r$ is control parameter $(0<r<1), m$ is a positive integer, and $g(m)$ is the $m$-th iteration of $g($.$) . Here we$ set $m=1$ since we want to stabilize the average queue size to a fixed point. According to Theorem 1 in [10], we can see that the fixed point of controlled system (9) is equal to that in original system (1). As an example, we investigate the controlled results when different parameters, such as the exponential averaging weight $w$, the maximum packet drop probability $p_{\max }$, the minimum and maximum thresholds $q_{\min }$ and $q_{\max }$, are varied.

\subsection{Exponential Averaging Weight}

Now we focus on the stable parameter range of controlled system. We represent the controlled system as

$$
\bar{q}_{k+1}=Q\left(\bar{q}_{k}, \rho\right)=\operatorname{rg}\left(\bar{q}_{k}, \rho\right)+(1-r) \bar{q}_{k}
$$

The eigenvalue of the controlled system is described as

$$
\left.\frac{\partial Q\left(\bar{q}_{k}, \rho\right)}{\partial \bar{q}_{k}}\right|_{\bar{q}_{k}=q^{*}}=r \frac{\partial g\left(\bar{q}_{k}, \rho\right)}{\partial \bar{q}_{k}}+1-\left.r\right|_{\bar{q}_{k}=q^{*}}
$$

Substituting (6) into (11) yields

$$
\left.\frac{\partial Q\left(\bar{q}_{k}, \rho\right)}{\partial \bar{q}_{k}}\right|_{\bar{q}_{k}=q^{*}}=r\left(1-w-w \frac{e}{2-k}\left(q^{*}-q_{\min }\right)^{\frac{k-3}{2-k}}\right)+1-r
$$

If the controlled system is stabilized to the fixed point, the linear stability criterion should be satisfied, i.e.

$$
\left|r\left(1-w-w \frac{e}{2-k}\left(q^{*}-q_{\min }\right)^{\frac{k-3}{2-k}}\right)+1-r\right|<1
$$

Therefore, the critical value of the controlled system is expressed in the following closed form according to inequality (13):

$$
w_{c h}=\frac{2}{r\left(1+\frac{e}{2-k}\left(q^{*}-q_{\min }\right)^{\frac{k-3}{2-k}}\right)}=\frac{1}{r} w_{c}
$$

Since the control parameter $r$ is valued in a range of $0<r<1$, the critical value $w_{c h}$ of controlled system is larger than $w_{c}$ of original system. That is to say, the stable range is extended in the controlled system. We vary $w$ from 0.0 to 0.5 . Other parameters are the same as those in Section 3 respectively. Here we set the 
control parameter $r=0.8$. The control result is shown in Fig. 3. We conclude that the first period-doubling bifurcation is delayed from $w_{c}=0.269$ (Fig. 1) to $w_{c h}=0.0336$ (Fig. 3). Meanwhile, the average queue size of controlled system is stabilized to the fixed point $q^{*}=338.3$ which is equal to that in original system. From the diagram we can see that the numerical simulation is consistent with the theoretical analysis.

According to inequality (13), we can also determine the stable range of control parameter in which the controlled system is stabilized to the fixed point. This range is expressed as:

$$
0<\alpha<\min \left\{1, \frac{2}{w\left(1+\frac{e}{2-k}\left(q^{*}-q_{\text {min }}\right)^{\frac{k-3}{2-k}}\right)}\right\}
$$

We set $w=0.28$ as an example for controlling chaos. From Fig. 2, we know that the system is in chaos when $w=0.28$. According to inequality (15), we obtain a stable range of control parameter $0<r<1$. Here we choose $r=0.8$ which is in its stable range. The control result is shown in Fig. 4. The hybrid control strategy is applied to the system at time 200 . It shows that the system is stabilized to the fixed point rapidly.

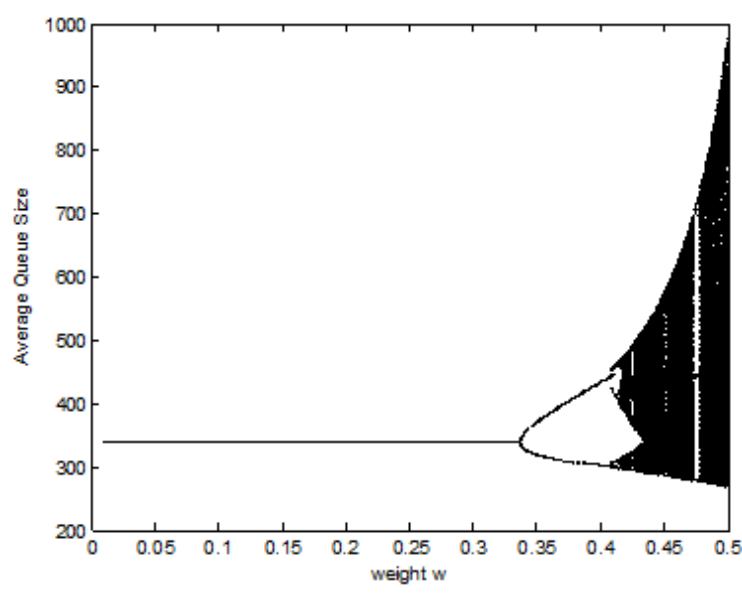

Fig. 3. Bifurcation Diagram with $w(r=0.8)$.

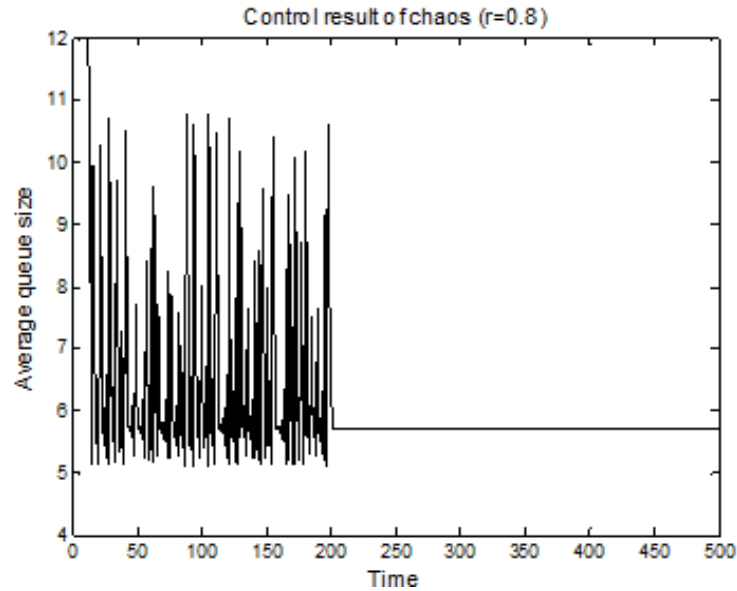

Fig. 4. Control result of chaos with $r=0.8$ and $w=0.28$.

\subsection{Drop Probability}

In this subsection, we choose the maximum packet drop probability $p_{\max }$ as the bifurcation parameter. $p_{\max }$ is varied from 0.02 to 0.18 . The exponential averaging weight $w$ is 0.28 . The corresponding bifurcation diagram is shown in Fig. 5, the control result of bifurcation with $r=0.8$ is shown in Fig. 6 .

Because the fixed point depends on the maximum packet drop probability $p_{\max }$, the controlled stable value varies with the bifurcation parameter. From Fig. 6, we can see that the chaotic behavior of system is eliminated completely when $p_{\max }$ is in the range 0.02 to 0.13 . However, the plot of fixed point before the first period doubling bifurcation is not a straight line as that in Fig. 1 because the fixed point is varying with changing $p_{\text {max }}$.

\subsection{Lower And Upper Threshold as Bifurcation Parameter}

In this subsection, how $q_{\min }$ affects system stability and behavior is studied. $q_{\min }$ is varied from 150 to 450 . The lower threshold $q_{\min }$ is varied from 150 packet to 450 packet and the upper threshold $q_{\max }=q_{\min }+500$ packets are varied whereas other parameters are fixed. The exponential averaging weight $\mathrm{w}$ is 0.28 . The 
corresponding bifurcation diagram is shown in Fig. 7, from which it can be seen that similar nonlinear behavior is exhibited in this case as well. The control result of bifurcation with $r=0.8$ is shown in Fig. 8, Because the fixed point depends on the lower and upper threshold $q_{\min }$, the controlled stable value varies with the bifurcation parameter. From Fig. 8, we can see that the chaotic behavior of system is eliminated completely when $q_{\min }$ is in the range 150 to 300 .

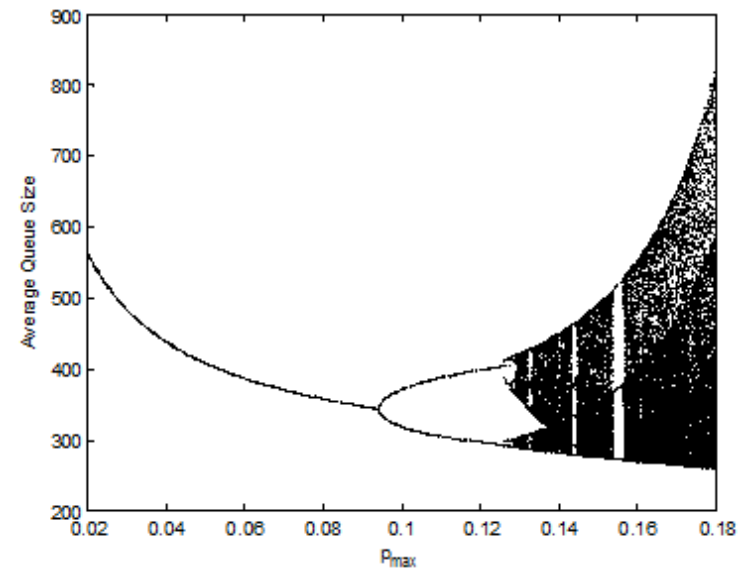

Fig. 5. Bifurcation diagram with $p_{\max }$.

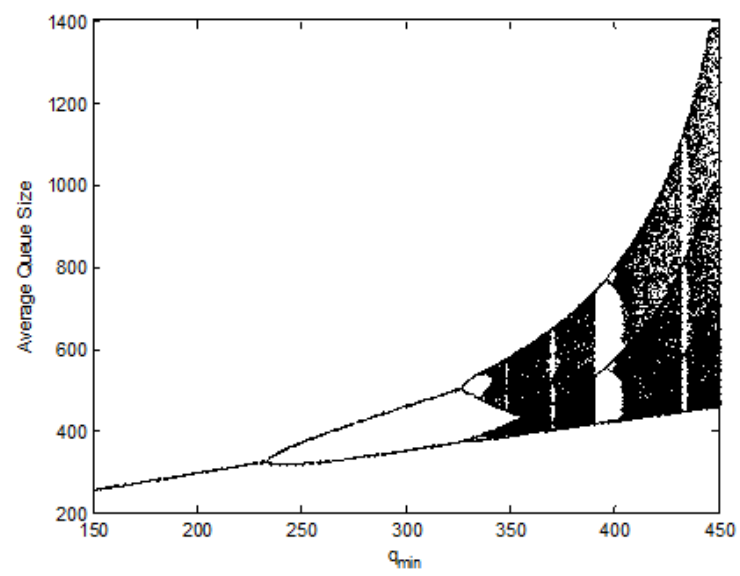

Fig. 7. Bifurcation diagram with $q_{\text {min. }}$

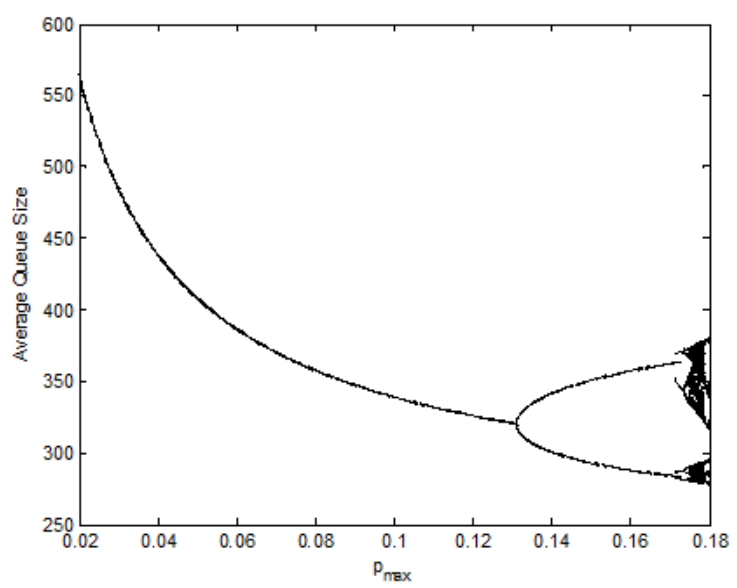

Fig. 6. Bifurcation diagram with $p_{\max }(r=0.8)$.

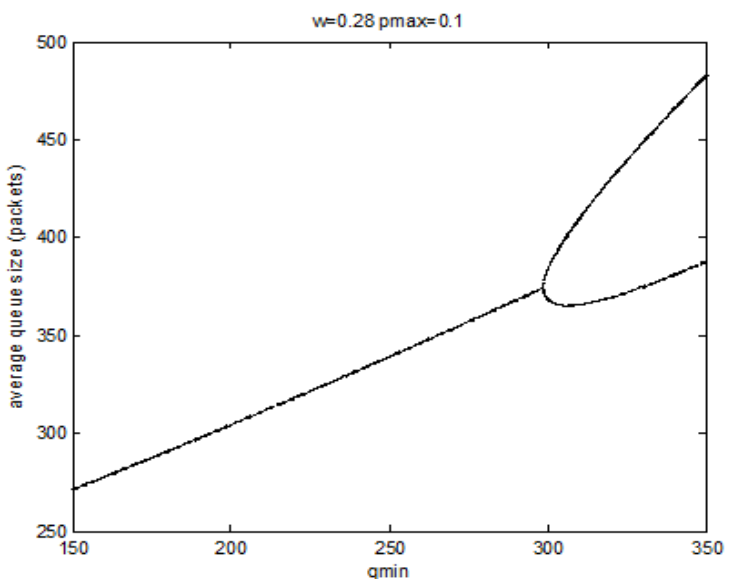

Fig. 8. Bifurcation diagram with $q_{\min }(r=0.8)$.

\subsection{Number of Connections}

Unlike the parameter studied above, the number of TCP connections $N$ and the propagation delay of the networks cannot be controlled by a network manager. Knowing the effect of the number of TCP connections $N$ and the propagation delay on system stability and behaviors is important for setting the RED control parameters in practice. In this subsection, we choose the number of TCP connections $N$ as the bifurcation parameter. $N$ is varied from 0.5 to 2 . The exponential averaging weight $w$ is 0.28 .

The corresponding bifurcation diagram is shown in Fig. 9. The control result of bifurcation with $r=0.8$ is shown in Fig. 10. Because the fixed point depends on the number of TCP connections $N$, the controlled stable value varies with the bifurcation parameter. From Fig. 10, we can see that the chaotic behavior of system is eliminated completely when $N$ is in the range 0.46 to 2 .

\subsection{Propagation Delay}

The effect of the propagation delay on system stability and behaviors is important for setting the RED 
control parameters in practice. In this subsection, we choose the number of TCP connections $d$ as the bifurcation parameter. $d$ is varied from 0.08 to 0.13 . The exponential averaging weight $w$ is 0.28 . The corresponding bifurcation diagram is shown in Fig. 9, The control result of bifurcation with $r=0.8$ is shown in Fig. 11, Because the fixed point depends on the of the propagation delay $d$, the controlled stable value varies with the bifurcation parameter. From Fig. 12, we can see that the chaotic behavior of system is eliminated completely when $N$ is in the range 0.08 to 0.12 .

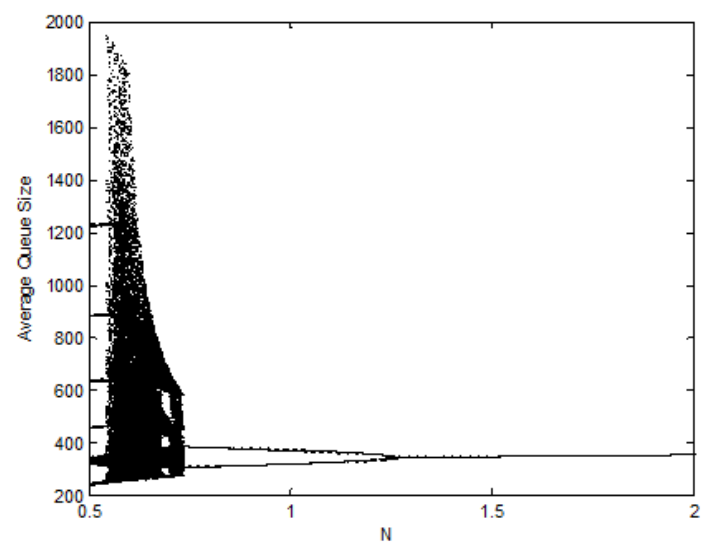

Fig. 9. Bifurcation diagram with $N$.

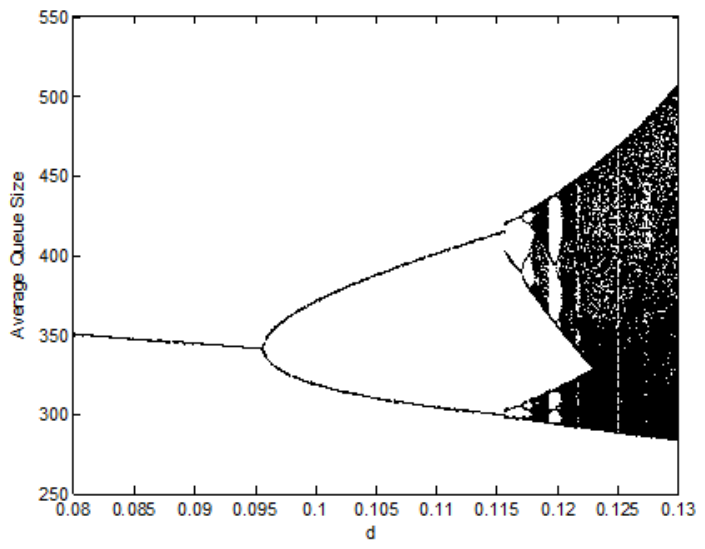

Fig. 11. Bifurcation diagram with $d$.

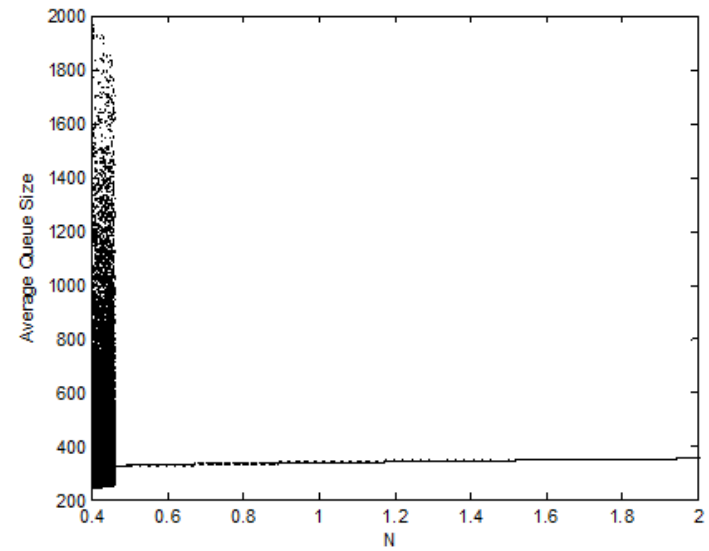

Fig. 10. Bifurcation diagram with $N(r=0.8)$.

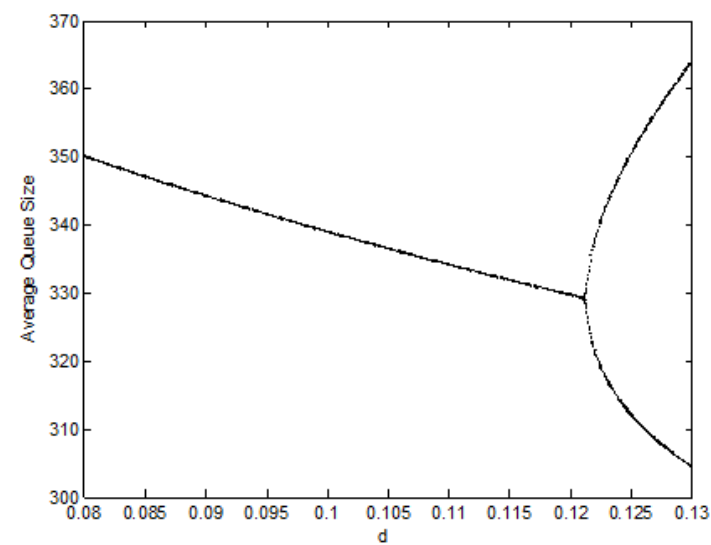

Fig. 12. Bifurcation diagram with $d(r=0.8)$.

\section{Conclusion}

In this paper, we first introduced a dynamic model of CTCP connections with RED gateway system and then analyzed its period-doubling bifurcation and chaotic behavior by bifurcation diagram and Lyapunov exponent diagram. Then, we have employed a hybrid control strategy by combining state feedback and parameter perturbation for controlling bifurcation and chaos in this model to extend the stable parameter range of TCP/RED. This hybrid control strategy does not use the exact fixed point or any system parameters for controlling. Theoretical analysis and numerical simulation results have demonstrated that it works effectively. Therefore, it is possible to extend the stable region in parameter space and improve the stability and performance of CTCP connections with RED gateway congestion control system. In the future, some novel and practical control strategies such as delayed feedback control approaches will be employed to control the system state parameter for controlling bifurcation and chaos in this model in order to extend the stable parameter range of TCP/RED [16] 


\section{Acknowledgment}

This paper is supported by the National Natural Science Foundation of China (No:61201227), College Students' innovation and entrepreneurship training plan of Anhui University (J18515089), College Student' research training plan of Anhui University (J18520170).

\section{References}

[1] Rao, A. (2013). Stability analysis of compound TCP with adaptive virtual queues. Proceedings of 25th Chinese Control and Decision Conference (pp. 2148-2154).

[2] Chavan, S., \& Raina, G. (2013). Dynamics of compound TCP with small buffer drop-tail queues. Proceedings of IEEE International Conference on Control Applications (pp. 691-697).

[3] Patel, C. M. (2013). URED: Upper threshold RED an efficient congestion control algorithm. Proceedings of Fourth International Conference on Computing, Communications and Networking Technologies (pp. 1-5).

[4] Zhang, H., Liu, M. J., Vukadinović, V., \& Trajković, L. (2005). Complex Dynamics in Communication Networks. Berlin: Springer Berlin Heidelberg.

[5] Zhang, S., Jian, X., \& Chung, K. W. (2015). On the stability and multi-stability of a TCP/RED congestion control model with state-dependent delay and discontinuous marking function. Communications in Nonlinear Science and Numerical Simulation, 22(1-3), 269-284.

[6] Tan, K., Song, J. M., Zhang, Q., \& Sridharan, M. A. (2006). Compound TCP approach for high-speed and long distance network. Proceedings of 25th International Conference on Computer Communications (pp. 1-12).

[7] Tomita, N., \& Valaee, S. (2012). Data uploading time estimation for CUBIC TCP in long distance networks. Computer Networks, 56(11), 2677-2689.

[8] Charbonneau, N., \& Vokkarane, V. M. (2011). Performance modeling of HS-RR-TCP over load-balanced optical burst-switched (OBS) networks. Optical Switching and Networking, 8(2), 116-128.

[9] Ha, S., Rhee, I., \& Xu, L. (2008). CUBIC: A new TCP-friendly high-speed TCP variant. ACM SIGOPS Operating Systems Review, 42(5), 64-74.

[10] Kliazovich, D., Granelli, F., \& Miorandi, D. (2006). TCP Westwood+ enhancement in high-speed long-distance networks. Proceedings of IEEE International Conference on Communications: Vol. 2 (pp. 710-715).

[11] Chen, L., Wang, X. F., \& Zheng, Z. H. (2004). Controlling bifurcation and chaos in Internet congestion control model. International Journal of Bifurcation and Chaos, 14(5), 1863-1876.

[12] Chen, L., Wang, X. F., \& Zheng, Z. H. (2004). Controlling chaos in Internet congestion control model. Chaos, Solitons \& Fractals, 21(1), 81-91.

[13] Luo, X. S., Chen, G., Wang, G., Fang, B. H., \& Qing, J. (2003). Hybrid control of period-doubling bifurcation and chaos in discrete nonlinear dynamical systems. Chaos, Solitons \& Fractals, 18(4), 775-783.

[14] Ding, D. W., Zhu, J., \& Luo, X. S. (2008). Hybrid control of bifurcation and chaos in stroboscopic model of internet congestion control system. Chinese Physics B, 17(1), 105-110.

[15] Prasad, S., \& Raina, G. (2014). Local Hopf bifurcation analysis of compound TCP with an exponential-RED queue management policy. Proceedings of 26th Chinese Control and Decision Conference (CCDC) (pp. 2588-2594).

[16] Ding, D. W., Qiu, S., Guo, F., Zhang, K., \& Xu, X. F. (2013). Controlling chaos in mixed TCP and UDP model. Journal of Computational Information Systems, 9(23), 9341-9346. 


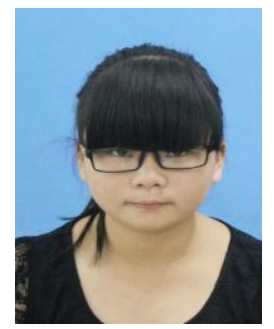

Mengya Geng was born in Anhui Province, China, in 1996. She is a junior student who is going to graduate in 2016 from Electronic and Information Engineering, Anhui University, Hefei, China.

She mainly engaged in the network systems and internet application development since 2012. She has attended in a provincial level research in July 2013. Her research interests include the complex dynamic networks, nonlinear dynamics and chaos, bifurcation.

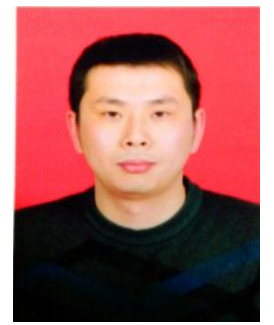

Dawei Ding works as an associate professor in the School of Electronics and Information Engineering of Anhui University, Hefei, China. He received the master's degree in circuits and systems from Anhui University in 2004, In 2008, he received the Ph.D. degree in circuits and systems from Shanghai Jiaotong University. He has held and participated in a number of national natural science fund researches. His research interests include communications networks, the nonlinear circuit network, the network congestion control, nonlinear dynamics and chaos, bifurcation.

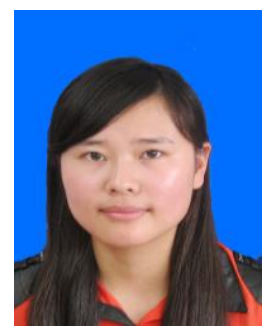

Han Wang was born in Anhui Province, China, in 1993. She is currently an undergraduate student of School of Electronics and Information Engineering of Anhui University, Hefei, China. Her research interests include the complex dynamic networks, nonlinear dynamics, congestion control, and chaos, bifurcation.

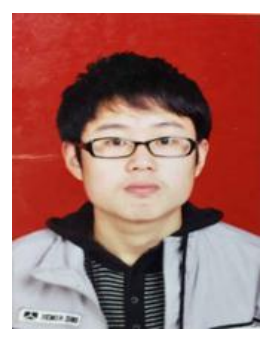

Jinyang Huang was born in Anhui Province, China, in 1994. He is a student in School of Electronics and Information Engineering of Anhui University, Hefei, China. His research interests include embedded system, nonlinear dynamics and chaos, bifurcation.

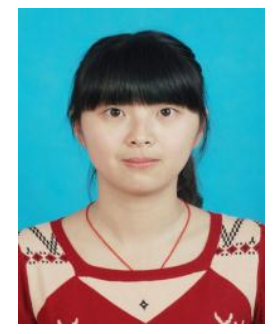

Jingwei Wang was born in Anhui Province, China, in 1994. She is an undergraduate student who will graduate in 2016 from the Department of Electronics and Information Engineering, Anhui University, Hefei, China. Her research interest include the complex dynamic networks, nonlinear dynamics and chaos, bifurcation.

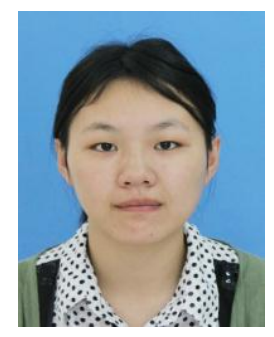

Haiyan Wang was born in Anhui Province, China, in 1994. She is currently a graduate student with electronic and information engineering, at the Department of Electronics and Information Engineering, Anhui University, Hefei, China. Her research interests include the complex dynamic networks, nonlinear dynamics and chaos, bifurcation. 